\title{
Long-term survival in a patient with metastatic squamous cell lung carcinoma: A case report
}

\author{
EMIDDIO BARLETTA ${ }^{1}$, PIERA FEDERICO ${ }^{1}$, VINCENZA TINESSA ${ }^{1}$, DOMENICO GERMANO ${ }^{1}$, \\ LUCIA CANNELLA ${ }^{1}$, TERESA PIRONTI ${ }^{2}$ and BRUNO DANIELE ${ }^{1}$ \\ ${ }^{1}$ Division of Medical Oncology and ${ }^{2}$ Department of Radiotherapy, 'G. Rummo' Hospital, I-82100 Benevento, Italy
}

Received February 16, 2017; Accepted August 2, 2017

DOI: $10.3892 /$ mco.2017.1408

\begin{abstract}
Non-small-cell lung cancer (NSCLC) is the most common malignancy in industrialized countries, with a 5-year survival rate of only $\sim 15 \%$, as the majority of the patients have advanced-stage disease at diagnosis and the treatment options are limited. Squamous cell carcinoma the second most frequent type of NSCLC and is closely associated with cigarette smoking. We herein present the case of a 72-year-old male smoker, diagnosed with stage IV squamous cell lung carcinoma, with a solitary brain metastasis. After the diagnosis, stereotactic radiotherapy was performed on the brain metastasis. Following radiotherapy, chemotherapy with carboplatin + paclitaxel was initiated. However, after 2 cycles of chemotherapy, disease progression in the lung was observed. Therefore, second-line treatment with pemetrexed was started, which was discontinued after 2 cycles due to further disease progression. Third-line treatment with erlotinib was then administered, with notable benefit, as the patient remains alive after 6 years of treatment with a good performance status. The mutation status of EGFR was unknown.
\end{abstract}

\section{Introduction}

Non-small-cell lung cancer (NSCLC) is the most common malignancy in industrialized countries, in incidence as well as mortality $(1,2)$. The probability of survival at 5 years, considering all-stage disease, remains low, at $\sim 15 \%$ Approximately $60-70 \%$ of the patients have advanced-stage disease at diagnosis, and the treatment options are limited $(3,4)$. The main cause of NSCLC is cigarette smoking, and the risk is directly proportional to the duration of exposure to smoking and the number of cigarettes smoked (5). Squamous cell carcinoma is the second most common type of NSCLC, and is closely associated with cigarette smoking. From a biological point

Correspondence to: Dr Emiddio Barletta, Division of Medical Oncology, 'G. Rummo' Hospital, 1 via dell'Angelo, I-82100 Benevento, Italy

E-mail: emiddiobarletta@libero.it

Key words: lung cancer, squamous carcinoma, erlotinib of view, smoking patients have different characteristics from non-smokers. Non-smokers develop a disease in which genetic abnormalities are more relevant, with tumor cell proliferation largely depending on epidermal growth factor receptor (EGFR). In smokers, however, EGFR is wild-type, whereas a mutation of the $\mathrm{K}$-ras oncogene is very common. We herein present the case of a 72-year-old male smoker, with stage IV (T3N1M1) squamous cell carcinoma of the lung, who was unresponsive to chemotherapy but responsive to tyrosine kinase inhibitor (TKI) treatment.

\section{Case report}

In July 2008, a 72-year-old male patient was diagnosed via CT-guided fine-needle aspiration biopsy with poorly differentiated squamous cell carcinoma of the inferior lobe of the left lung, with a maximum diameter of $53 \mathrm{~mm}$. The patient also presented with ipsilateral hilar lymphadenopathy (Fig. 1) and a 30-mm solitary brain metastasis of the right frontal lobe (Fig. 2). The conclusive diagnosis was stage IV squamous lung cell carcinoma (cT3cN1cM1). In September 2008, stereotactic radiotherapy was performed on the brain metastasis, at a dose of 25 Gy. In October 2008, chemotherapy was initiated, with carboplatin (area under the curve 5) and paclitaxel $175 \mathrm{mg} / \mathrm{m}^{2}$ on day 1 , every 21 days.

The patient received 2 cycles of chemotherapy, with considerable toxic effects, including National Cancer Institute (NCI) grade 3 peripheral neuropathy, grade 3 leukopenia, and grade 3 asthenia. The treatment was discontinued due to unacceptable levels of toxicity. In December 2008, second-line chemotherapy was initiated, with pemetrexed $500 \mathrm{mg} / \mathrm{m}^{2}$ on day 1, every 21 days. Two cycles were administered prior to treatment discontinuation, due to disease progression (the lung lesion increased to a maximum diameter of $80 \mathrm{~mm}$ ). In February 2009, third-line treatment with erlotinib $150 \mathrm{mg}$ /day was initiated. After 2 weeks of treatment, a grade 3 skin rash developed. The treatment was discontinued for 10 days, during which time the rash was managed with sulfosalicylate-based moisturizing ointments and creams, and oral minocycline at a dose of $100 \mathrm{mg} /$ day (improvement of skin rash to grade 1). The skin rash regressed to grade 1-2 and erlotinib is readministered at a reduced dose of $100 \mathrm{mg} /$ day. During the first 3 months of treatment, the patient suffered repeated exacerbations of the skin rash, reaching grade 3 in intensity, leading to periods 


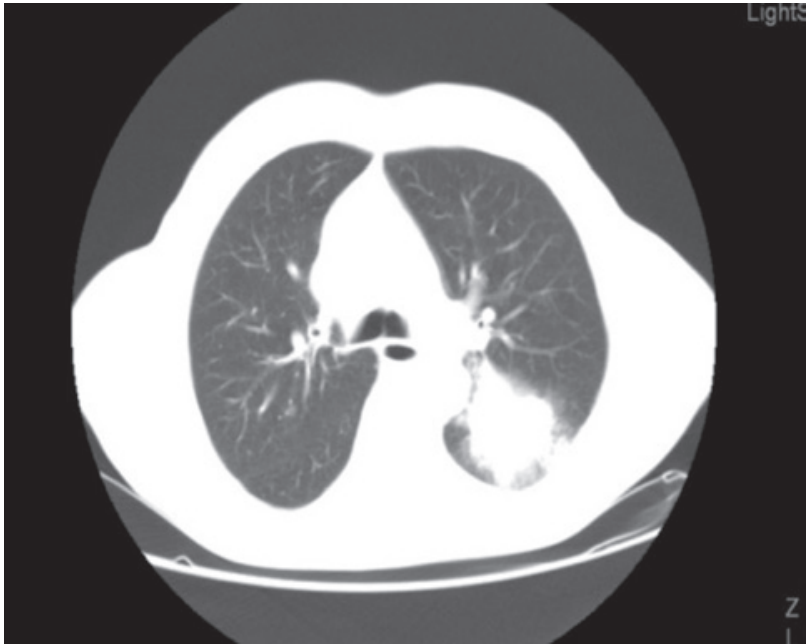

Figure 1. Computed tomography scan of the chest prior to erlotinib treatment, showing a 53-mm lesion in the inferior lobe of the left lung.

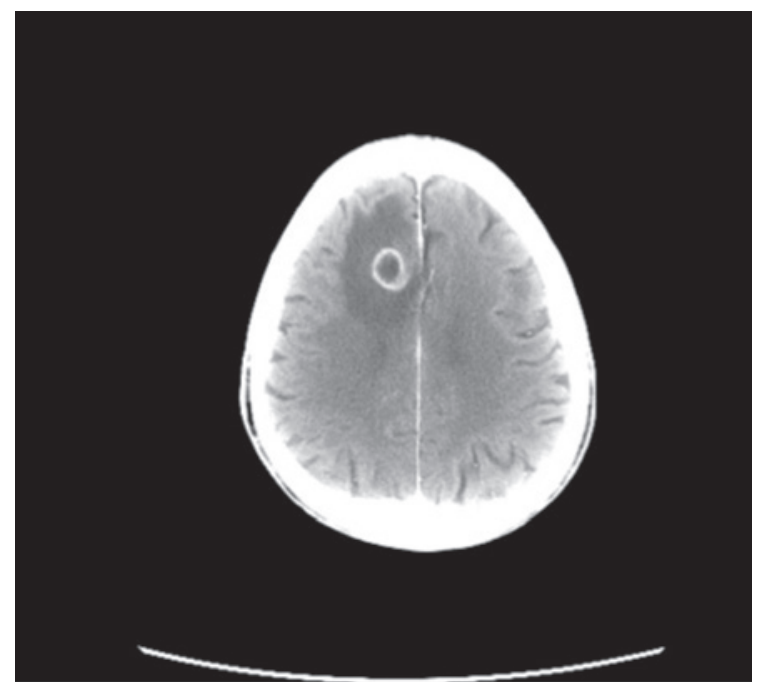

Figure 2. Computed tomography scan of the brain prior to erlotinib treatment, showing a $30-\mathrm{mm}$ metastatic lesion in the left frontal lobe.

of erlotinib discontinuation for 2 weeks. Three months later (May 2009), a whole-body computed tomography (CT) scan revealed a reduction in the size of the lung lesion from 80 to $42 \mathrm{~mm}$, with stability of the brain metastasis and no new disease locations, which was classed as a partial response according to the Response Evaluation Criteria In Solid Tumors (https://ctep.cancer.gov/protocoldevelopment/docs/ recist_guideline.pdf). The patient continued treatment with erlotinib $100 \mathrm{mg} /$ day, undergoing a whole-body CT scan every 3 months. The predominant toxic effect was skin rash, which varied between grade 1 and 2 over the years. For a total of 78 months (February 2009-September 2015), the patient was treated with erlotinib $100 \mathrm{mg} / \mathrm{day}$ and was planned to receive this treatment unless disease progression occurred. At the last whole-body CT scan, the size of the lung lesion had been reduced to $32 \mathrm{~mm}$ (Fig. 3) and the brain metastasis had been reduced to $20 \mathrm{~mm}$ (Fig. 4). The patient maintained an Eastern Cooperative Oncology Group performance status of 1 and a good quality of life. It should be noted that, in the present case,

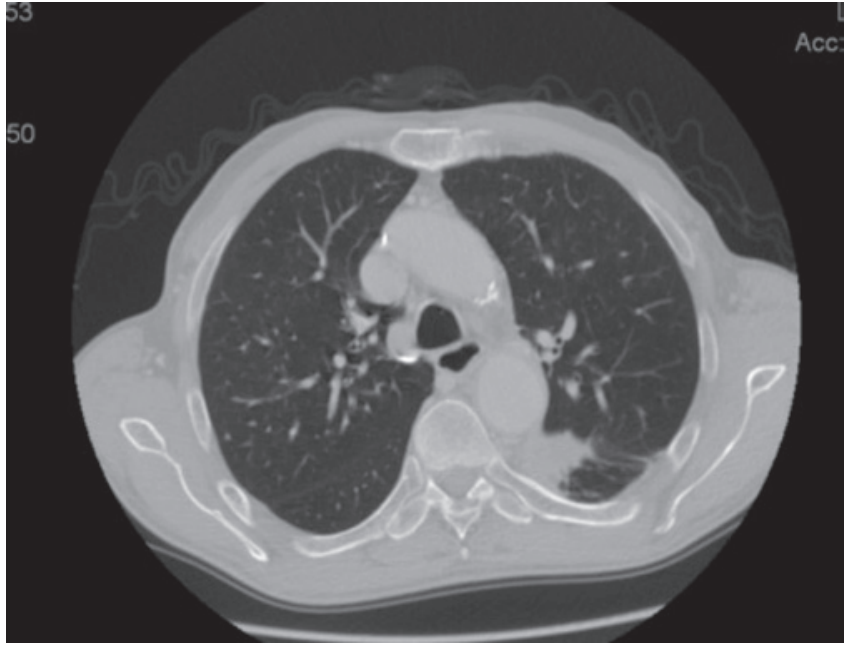

Figure 3. Computed tomography scan of the chest after 6 years of treatment with erlotinib, showing a significant reduction in the size of the lung primary.

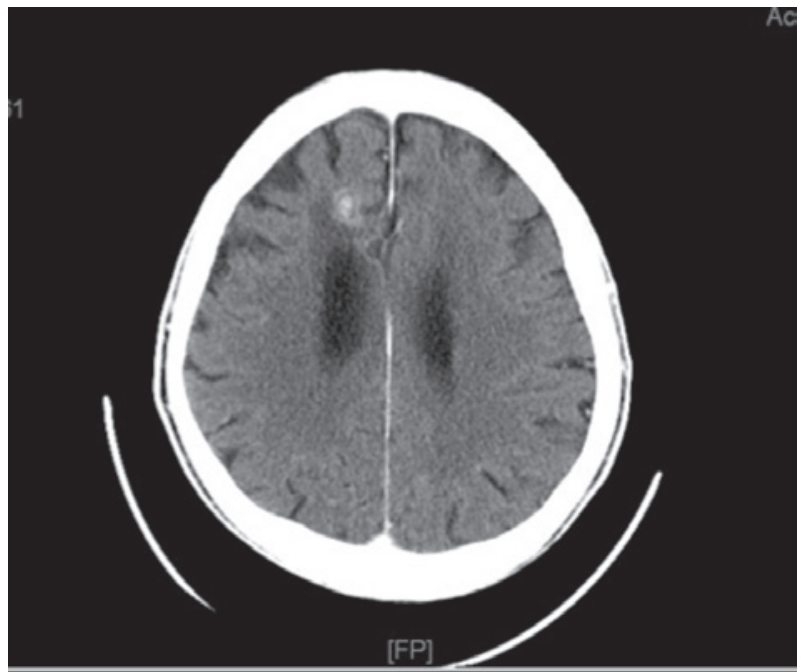

Figure 4. Computed tomography scan of the brain after 6 years of treatment with erlotinib, showing a significant reduction in the size of the brain metastasis.

the EGFR mutation status was unknown, as an examination was never performed due to insufficient cytological material. In October 2015 the patient died from causes unrelated to cancer (road traffic accident).

The patient provided informed consent to the publication of the case details and associated images.

\section{Discussion}

Squamous cell carcinoma of the lung, which comprises $25 \%$ of all NSCLC cases, is closely associated with cigarette smoking. The probability of finding activating mutations (codons 19-21 of the EGFR gene) in squamous cell carcinoma is relatively low ( $<3$ vs. $15 \%$ in adenocarcinomas). The presence of EGFR mutations is a predictor of response to TKI treatment (gefitinib and erlotinib). Erlotinib is a TKI of EGFR and is currently approved for the treatment of metastatic NSCLC after failure of at least one prior chemotherapy regimen, as maintenance therapy in patients with stable disease after 
4 cycles of standard first line platinum-based chemotherapy, and as a first-line treatment in the presence of activating EGFR mutations. The indication for second- and third-line treatment is based on data from the BR.21 (6) study, which compared erlotinib vs. best supportive care in an unselected population, showing an increase in overall survival (OS) with erlotinib (6.7 vs. 4.7 months).

The factors predictive of response to erlotinib are as follows: Adenocarcinoma histology, female gender, non-smoker status and Asian ethnicity. These factors were not found to be predictive of survival, however, as nearly all patients benefit from treatment with this drug, including smokers (6), although it should be remembered that cigarette smoking reduces exposure to erlotinib by $50-60 \%$. Another predictive factor investigated is the intensity of the rash: In exploratory analyses conducted in the BR.21 (6), SATURN (7), TITAN (8) and EURTAC (9) studies, it was observed that a higher grade of rash is associated with greater benefit in terms of progression-free survival (PFS) or OS. This assertion, however, required further confirmation by prospective studies designed ad hoc. In tumours characterized by the presence of mutations in codons 19-21 of the tyrosine kinase domain of EGFR (10), EGFR TKIs are associated with a high rate of objective response, PFS and OS (11). Erlotinib, in particular, has two positive phase III trials in this setting, one on Chinese and one on Caucasian patients $(9,12)$. The clinical case under consideration is characterized by the fact that the patient did not have the clinical characteristics considered predictive of response to the drug, as he was male, a smoker and had squamous cell carcinoma histology. Our patient was 'refractory' to chemotherapy, as he exhibited rapid progression of the disease; however, the selection of erlotinib as second line treatment has resulted in an excellent response in the lung and a reduction of the brain lesion after 3 months of treatment (13). The effectiveness of the drug was accompanied in the first 3 months by an intense skin rash located on the face, in the upper lip and periorbital region, which, however, subsided after 2-3 weeks and stabilized at grade 1. Skin rash is a frequent adverse event with erlotinib; it is, however, manageable by dose reduction and topical application of moisturizing creams, or administration of antibiotics in the case of bacterial superinfection. Correctly informing and monitoring the patient is a crucial element of therapy with erlotinib.

The results of the clinical case under consideration are encouraging, not only in terms of the objective response, but also in terms of quality of life and survival. The patient remained alive 72 months after initiation of treatment with erlotinib, and maintained a PS ECOG of 1. Although his EGFR mutation status was unknown, it is hypothesized to be positive, associated with other biomolecular factors predictive of positive response. The PFS with erlotinib in the EURTAC (9), SLCG (14), CALGB30406 (15) and OPTIMAL (12) studies ranges from 9.7 to 15.7 months. In the present case, at 72 months of therapy with erlotinib, there has been no evidence of disease progression. Our patient, while not having factors predicting response to TKIs (adenocarcinoma, non-smoker, female, Asian) exhibited a atypical, excellent response and long-term survival. In conclusion, it may be stated that the survival data reported in the clinical case examined herein are surprising and go beyond the current statistics.

\section{References}

1. Jemal A, Bray E, Center M, Ferlay J, Ward E and Forman D: Global cancer statistics. CA Cancer J Clin 61: 69-90, 2011.

2. Sant M, Allemani C, Santaquilani M, Knijn A, Marchesi F and Capocaccia R; EUROCARE Working Group. EUROCARE-4: Survival of cancer patients diagnosed in 1995-1999. Results and commentary. Eur J Cancer 45: 931-91, 2009.

3. Spiro SG and Silvestri GA: Oive hundred years of lung cancer. Am J Respir Crit Care Med 172: 523-529, 2005.

4. Youlden DR, Cramb SM and Baade PD: The International epidemiology of lung cancer: Geographical distribution and secural trends. J Thorac Oncol 3: 818-831, 2008.

5. Alberg AJ and Samet JM: Epidemiology of lung cancer. Chest 123 (1 Suppl): S21-S49, 2003.

6. Shepherd FA, Rodrigues Pereira J, Ciuleanu T, Tan EH, Hirsh V, Thongprasert S, Campos D, Maoleekoonpiroj S, Smylie M, Martins R, et al: Erlotinib in previously treated non-small-cell lung cancer. N Engl J Med 353: 123-132, 2005.

7. Neal JW: The SATURN trial: The value of maintenance erlotinib in patients with non-small-cell lung cancer. Future Oncol 6: 1827-1832, 2010.

8. Ciuleanu T, Stelmakh L, Cicenas S, Miliauskas S, Grigorescu AC, Hillenbach C, Johannsdottir HK, Klughammer B and Gonzalez EE: Efficacy and safety of erlotinib versus chemotherapy in second-line treatment of patients with advanced, non-small-cell lung cancer with poor prognosis (TITAN): A randomized multicentre, open-label, phase 3 study. Lancet Oncol13: 300-308, 2012.

9. Rosell R, Carcereny E, Gervais R, Vergnenegre A, Massuti B, Felip E, Palmero R, Garcia-Gomez R, Pallares C, Sanchez JM, et al: Erlotinib versus standard chemotherapy as first-line treatment for patients with advanced EGFR mutation-positive non-small-cell lung cancer (EURTAC): A multicentre, open-label, randomized phase 3 study. Lancet Oncol 13: 239-246, 2012.

10. Johnson BE and Jänne PA: Epidermal growth factor receptor mutations in patients with non-small cell lung cancer. Cancer Res 65: 7525-7529, 2005.

11. Jackman DM, Yeap BY, Sequist LV, Lindeman N, Holmes AJ, Joshi VA, Bell DW, Huberman MS, Halmos B, Rabin MS, et al: Exon 19 deletion mutations of epidermal growth factor receptor are associated with prolonged survival in non-small cell lung cancer patients treated with gefitinib or erlotinib. Clin Cancer Res 12: 3908-3914, 2006.

12. Zhou C, Wu YL, Chen G, Feng J, Liu XQ, Wang C, Zhang S, Wang J, Zhou S, Ren S, et al: Erlotinib versus chemotherapy as first-line treatment for patients with advanced EGFR mutation-positive non small-cell lung cancer (OPTIMAL, CTONG-0802): A multicentre, open label, randomised, phase 3 study. Lancet Oncol. 12: 735-742, 2011.

13. Popat S, Hughes S, Papadopoulos P, Wilkins A, Moore S, Priest K, Meehan L, Norton A and O'Brien M: Recurrent responses to non-small cell lung cancer brain metastases with erlotinib. Lung Cancer 56: 135-137, 2007.

14. Grace Li: TLCR is endorsed by the Spanish Lung Cancer Group (SLCG): New horizons for strong academic collaboration in lung cancer J Thorac Dis. Dec; 5: E217-E218, 2013.

15. Rosell R, Carcereny E, Gervais R, Vergnenegre A, Massuti B, Felip E, Palmero R, Garcia-Gomez R, Pallares C, Sanchez JM, et al: Erlotinib versus standard chemotherapy as first-line treatment for patients with advanced EGFR mutation-positive non-small-cell lung cancer (EURTAC): A multicentre, open-label, randomized phase 3 study. Lancet Oncol 13: 239-246, 2012. 